\title{
Three-dimensional position and velocity measurements using a pair of linear-period-modulated ultrasonic waves
}

\author{
Natee Thong-un ${ }^{1, *}$, Shinnosuke Hirata ${ }^{2}$, Minoru K. Kurosawa ${ }^{1}$ and Yuishiro Orino ${ }^{1}$ \\ ${ }^{1}$ Department of Information Processing, Interdisciplinary Graduate School of Science and Engineering, \\ Tokyo Institute of Technology, G2-32, 4259 Nagatsuta, Midori-ku, Yokohama, 226-8502 Japan \\ ${ }^{2}$ Department of Mechanical and Control Engineering, Graduate School of Science and Engineering, \\ Tokyo Institute of Technology, S5-17, 2-12-1, Ookayama, Meguro-ku, Tokyo, 152-8552 Japan
}

( Received 31 October 2012, Accepted for publication 24 December 2012)

Keywords: Linear-period-modulated signal, Single-bit signal processing, Three-dimensional ultrasonic system PACS number: 43.58.+z, 43.60.+d [doi:10.1250/ast.34.233]

\section{Introduction}

Nowadays, it is necessary for most autonomous mobile robots to recognize the distance between their own location and obstacles. In detection methods employing acoustical systems, proposed in several papers [1-4], ultrasonic waves are used because of easy reflection from structures. The advantages of ultrasonic systems, compared with others, are low cost, small size, and simple hardware. For these reasons, ultrasonic distance measurement is the most up-to-date and reliable for environment recognition by autonomous mobile robots. The pulse-echo method is one of the general methods of such measurement. It relies on the determination of the time-of-flight (TOF) of an echo reflected [4]. In addition, pulse compression has been introduced to the pulse-echo method for improving the signal-to-noise ratio (SNR) of the received signal and distance resolution [5].

A linear-frequency-modulated (LFM) signal, which indicates the change in instantaneous frequency with time, has much greater potential in sonar, radar, and ultrasonic applications, for example. TOF can readily be computed from transmitted LFM and echo signals and is identified by the maximum peak in the cross-correlation function from these signals. However, the cross-correlation function has many iterations of multiplications and summations in signal processing. Because of the high computational-time cost, it is inappropriate for application to digital signal processing. Therefore, a method using delta-sigma modulated single-bit signal processing has been proposed to reduce the number of routines in the cross-correlation function [5].

However, the LFM signal is effectively modulated by the Doppler effect, which is unsuitable for the case of moving objects. For this reason, a Doppler-shifted LFM signal is not capable of complete correlation with a transmitted reference LFM signal. To satisfy this problem, a linear-period-modulated (LPM) signal, a period of which is linearly swept with time, has been presented for ultrasonic systems of moving objects $[6,7]$. It enables the cross-correlation function possible to yield the TOF from the reflected echo. Although this may be develop a solution to overcome the Doppler effect, it still requires much time because of the use of the envelop-signal

*e-mail: thnatee@yahoo.co.th calculation. Thus, a low-calculation-cost method of ultrasonic measurement by pulse compression comprising two cycles of LPM signals and Doppler-shift compensation has been proposed [8]. Accordingly, to achieve fast signal processing, we have applied such a method.

Ultrasonic distance and velocity measurements have already been accomplished by using one microphone [8]. In the case of an ultrasonic two-dimensional system, a solution for two-dimensional position and velocity measurements by exploiting two microphones has also been published [9]. Nevertheless, the development of position and velocity measurement under ultrasonic system is far from complete. Consequently, to make them applicable to three-dimensional systems, we propose a simple method for estimating the position and velocity of a single moving object using three microphones in Euclidean space. In contrast to sound-source localization, these techniques were designed to estimate the object position using four receivers $[10,12]$.

In [8], the method of Doppler-effect compensation to measure TOF is described; the Doppler velocity is used for each microphone on $X, Y$, and $Z$ axes. Because this designed system is made up of nonlinear equations, Taylor-series expansion is directly adopted. A set of nonlinear equations is linearized by Taylor-series expansion to estimate a target point $[13,14]$. To keep the method simple, we use only the first two terms of Taylor-series expansion, namely, the Newton-Raphson method. After already obtaining Dopplervelocity values and an object-position value from three microphones, a formula for identifying the 3-D velocity of a moving object, which is based on vector theory, is also proposed. Simulation results show that results are in good agreement with the proposed system.

\section{Object-position estimation}

The ultrasonic three-dimensional system proposed in this paper is shown in Fig. 1. The devices displayed under the designed system mainly consist of a speaker and three microphones. A transmitted signal exciting to the speaker employs a pair of two LPM signals. An echo signal from the moving object is incident on microphones and converted to a digital signal by three delta-sigma modulators. On the other hand, a reference signal, which is the LPM signal, utilizes a 


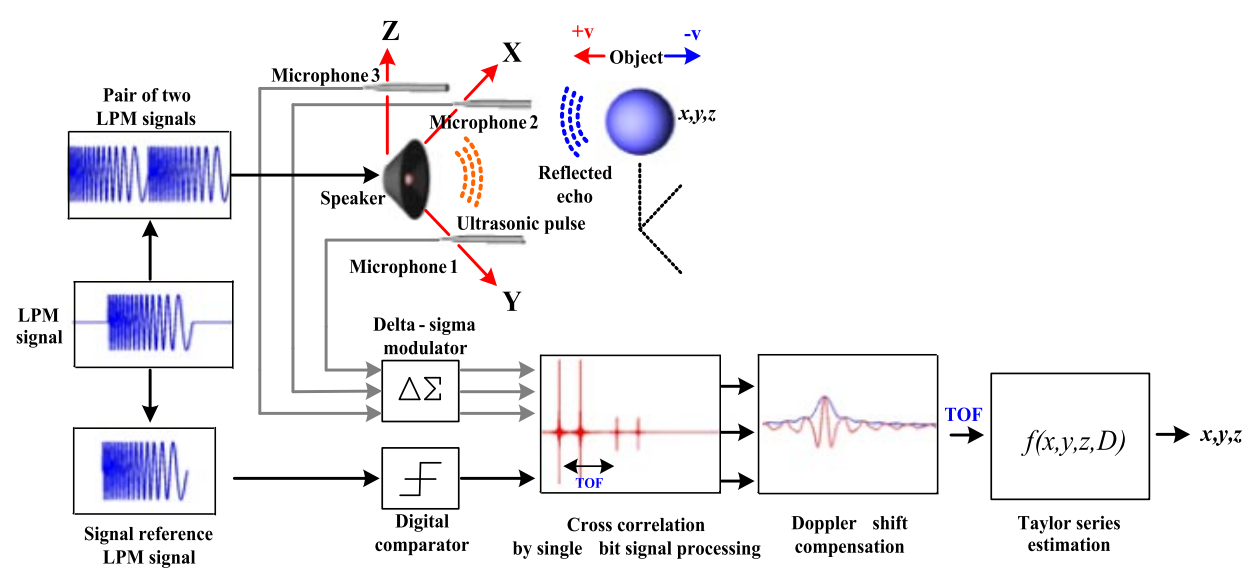

Fig. 1 Proposed ultrasonic three-dimensional system.

digital comparator as a signal converter. Then, both digital signals from those devices are processed together to compute TOF by means of the cross-correlation function. Single-bit signal processing described in $[2,8]$ plays a vital part in saving computational-time cost. In the case of non-Doppler effect, TOF of a received LPM signal is typically estimated from the maximum peak time in the cross-correlation function. Unfortunately, in the case of the moving object, the crosscorrelation function for TOF estimation of the received LPM signal is shifted owing to the Doppler effect. To overcome this effect, TOF is technically estimated by performing the Doppler-shift compensation from the maximum and minimum peaks of the cross-correlation function [8]. When considered under Euclidean geometry, as in Fig. 1, equations created by referring to the relationship between the object, speaker, and microphone locations, and TOF are

$$
\begin{aligned}
& f_{1}=\sqrt{x^{2}+y^{2}+z^{2}}-D=0 \\
& f_{2}=\sqrt{\left(x-x_{1}\right)^{2}+y^{2}+z^{2}}+D-c \cdot \text { TOF }_{x}=0 \\
& f_{3}=\sqrt{x^{2}+\left(y-y_{2}\right)^{2}+z^{2}}+D-c \cdot \text { TOF }_{y}=0 \\
& f_{4}=\sqrt{x^{2}+y^{2}+\left(z-z_{3}\right)^{2}}+D-c \cdot \text { TOF }_{z}=0
\end{aligned}
$$

where $x, y$, and $z$ describe the unknown position of the moving object, $D$ is the unknown distance between the speaker and the object, $x_{1}, y_{2}$, and $z_{3}$ are microphone positions along $X, Y$, and $Z$ axes, $T O F_{x}, T O F_{y}$, and $T O F_{z}$ are times-of-flight in each microphone, calculated from the Doppler-shift compensation technique, and $c$ is the sound velocity in air. Basically, to solve the proposed-equations problem, Taylor-series estimation, having an easy simulation and readily tested convergence [14], is effective in analyzing the nonlinear problem efficiently. In this paper, Taylor-series estimation, sometimes called the Newton-Raphson method, plays an important role in the identification of the object position or $x, y$, and $z$ variables. The formula for four unknown parameters established in this paper is

$$
\nabla \boldsymbol{F} \cdot \delta=-f
$$

where

$$
\begin{gathered}
\nabla \boldsymbol{F}=\left[\begin{array}{cccc}
\frac{\partial f_{1}}{\partial x} & \frac{\partial f_{1}}{\partial y} & \frac{\partial f_{1}}{\partial z} & \frac{\partial f_{1}}{\partial D} \\
\frac{\partial f_{2}}{\partial x} & \frac{\partial f_{2}}{\partial y} & \frac{\partial f_{2}}{\partial z} & \frac{\partial f_{2}}{\partial D} \\
\frac{\partial f_{3}}{\partial x} & \frac{\partial f_{3}}{\partial y} & \frac{\partial f_{3}}{\partial z} & \frac{\partial f_{3}}{\partial D} \\
\frac{\partial f_{4}}{\partial x} & \frac{\partial f_{4}}{\partial y} & \frac{\partial f_{4}}{\partial z} & \frac{\partial f_{4}}{\partial D}
\end{array}\right], \\
\delta=\left[\begin{array}{c}
x-x_{0} \\
y-y_{0} \\
z-z_{0} \\
D-D_{0}
\end{array}\right), f=\left(\begin{array}{c}
f_{2} \\
f_{3} \\
f_{4}
\end{array}\right)
\end{gathered}
$$

where $x_{0}, y_{0}, z_{0}$, and $D_{0}$ are initial values, and $\delta$ is update data. However, this iterative method has significant disadvantage, that is, the requirement of an initial guess [13,14]. When carefully scrutinizing all TOFs measured from microphones, we enable them to be used as a starting point of routines. To do so, an initial distance $\left(D_{0}\right)$ is averaged by the travelling time, for progressing only one way from the speaker to the object, and thereafter multiplied with sound velocity.

$$
D_{0}=\frac{c \cdot\left(T O F_{x}+T O F_{y}+T O F_{z}\right)}{2 \times 3}
$$

From Eq. (1), assume that $x_{0}, y_{0}$, and $z_{0}$ have the same values. The initial object position can be determined easily, as below. Therefore, the obtained initial values are dependent on the TOF of the moving object.

$$
x_{0}=y_{0}=z_{0}=\frac{D_{0}}{\sqrt{3}}
$$

In every time instance in which the update data are computed, data are added with the old values for the new values.

$$
\delta^{k}=-\left[\nabla \boldsymbol{F}^{k}\right]^{-1} \cdot f^{k}
$$

After that, update and check the convergence criterion; stop when the difference is less than the tolerance, $\varepsilon$. 


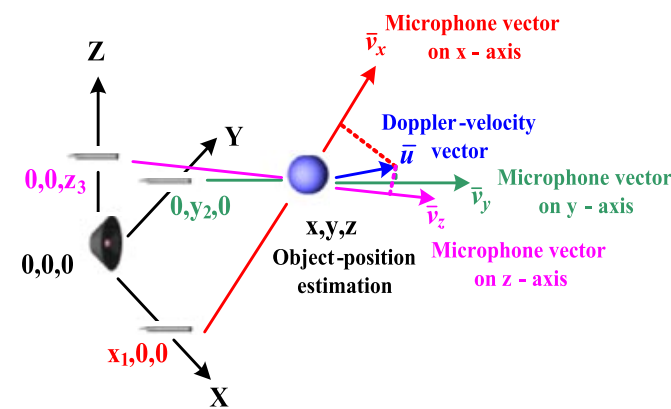

Fig. 2 Object-velocity vector configuration.

$$
\left(\begin{array}{c}
x \\
y \\
z \\
D
\end{array}\right)^{k+1}=\left(\begin{array}{c}
x \\
y \\
z \\
D
\end{array}\right)^{k}+\delta^{k} ;\left|\left(\begin{array}{c}
x \\
y \\
z \\
D
\end{array}\right)^{k+1}-\left(\begin{array}{c}
x \\
y \\
z \\
D
\end{array}\right)^{k}\right|<\varepsilon
$$

\section{Object-velocity estimation}

In the next step, the moving-object velocity is estimated using data of the Doppler-shift velocity, the instantaneous position of the moving object, and microphone positions. On the based of the fundamentals of vector theory, vector projection can be thought of as the dot product between two vectors shown to Fig. 2. Hence, the results of vector projection between the object-velocity and microphone vectors are exactly the Doppler-shifted velocity estimation at each microphone, depicted as

$$
\left(\begin{array}{c}
v_{d x} \\
v_{d y} \\
v_{d z}
\end{array}\right)=\boldsymbol{u} \cdot\left(\begin{array}{c}
\frac{\boldsymbol{v}_{x}}{\left|\boldsymbol{v}_{x}\right|} \\
\frac{\boldsymbol{v}_{y}}{\left|\boldsymbol{v}_{y}\right|} \\
\frac{\boldsymbol{v}_{z}}{\left|\boldsymbol{v}_{z}\right|}
\end{array}\right)
$$

$v_{d x}, v_{d y}$, and $v_{d z}$ are the Doppler-shifted velocity estimation at all microphones. $\boldsymbol{u}$ is the unknown object-velocity vector. $\boldsymbol{v}_{x}$, $\boldsymbol{v}_{y}$, and $\boldsymbol{v}_{z}$ are the microphone vectors.

$$
\boldsymbol{u}=\left(\begin{array}{c}
u_{x} \\
u_{y} \\
u_{z}
\end{array}\right) ; \boldsymbol{v}_{x}=\left(\begin{array}{c}
x-x_{1} \\
y \\
z
\end{array}\right) ; \boldsymbol{v}_{y}=\left(\begin{array}{c}
x \\
y-y_{2} \\
z
\end{array}\right)
$$

and

$$
\boldsymbol{v}_{z}=\left(\begin{array}{c}
x \\
y \\
z-z_{3}
\end{array}\right)
$$

Substitute Eq. (9) into Eq. (8) and rearrange again.

$$
\left(\begin{array}{l}
u_{x} \\
u_{y} \\
u_{z}
\end{array}\right)=\left[\begin{array}{ccc}
x-x_{1} & y & z \\
x & y-y_{2} & z \\
x & y & z-z_{3}
\end{array}\right]^{-1} \cdot\left(\begin{array}{c}
v_{d x} \cdot\left|\boldsymbol{v}_{x}\right| \\
v_{d y} \cdot\left|\boldsymbol{v}_{y}\right| \\
v_{d z} \cdot\left|\boldsymbol{v}_{z}\right|
\end{array}\right)
$$
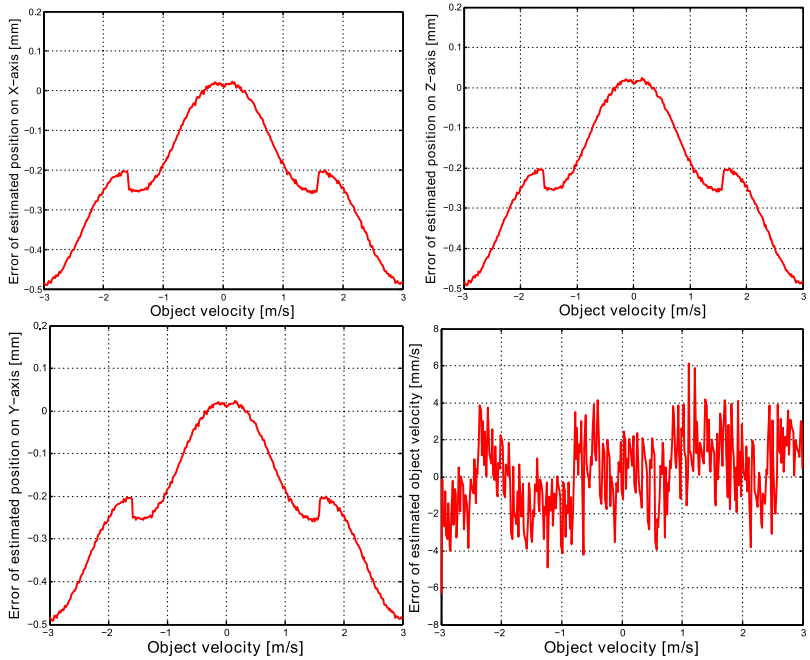

Fig. 3 Errors of object position and velocity measurements at an instantaneous position $x=y=z=1 \mathrm{~m}$.

By inserting a solution of Eq. (10) back into Eq. (9), the object-velocity vector $\boldsymbol{u}$ can simply be determined. Also, the magnitude of object-velocity estimation can be expressed as

$$
|\boldsymbol{u}|=\sqrt{u_{x}^{2}+u_{y}^{2}+u_{y}^{2}}
$$

\section{Evaluation of proposed ultrasonic three-dimensional system}

The object position and velocity estimation under an ultrasonic three-dimensional system determined by the proposed signal processing method were evaluated by MATLAB computer simulation. The period of the single LPM signal was swept linearly from $20 \mu$ s to $50 \mu$ s. The sampling frequency rate was $50 \mathrm{MHz}$. The distance from the speaker to each microphone was $10 \mathrm{~cm}$. In the environment setup, the LPM signal had the length of a pair of LPM signals equal to $6.548 \mathrm{~ms}$. The propagation velocity of an ultrasonic wave in air was $331.5 \mathrm{~m} / \mathrm{s}$. SNR was set at $0 \mathrm{~dB}$ compared with the amplitude of the echo signals, and the constant attenuation factor of the received signal was 0.1 times degraded from that of the original signal. In simulation, the Doppler velocity of the moving object was varied from -3 to $3 \mathrm{~m} / \mathrm{s}$. For each Doppler velocity, the object position was evaluated by 100 simulations. The probability distributions of the determined object position, under the assumption that there constantly was an instantaneous position, were set up at $x=y=z=1 \mathrm{~m}$ in Fig. 4. The moving-object-position errors varying with the object velocity are displayed in the top and bottom onto left side of Fig. 3. The graphs are not smooth at about $\pm 1.5 \mathrm{~m} / \mathrm{s}$ because the waveform of the cross-correlation function, based on Doppler shift, is inverted at those points. The errors due to Doppler-shift compensation are sharper in such case. In the bottom right of Fig. 3, it shows the deviation of objectvelocity estimation when the object was continuously moving at velocity from -3 to $3 \mathrm{~m} / \mathrm{s}$. Figure 5 illustrates the movingobject direction with its component vectors computed using Eq. (10). In this case, the directions had been assumed 

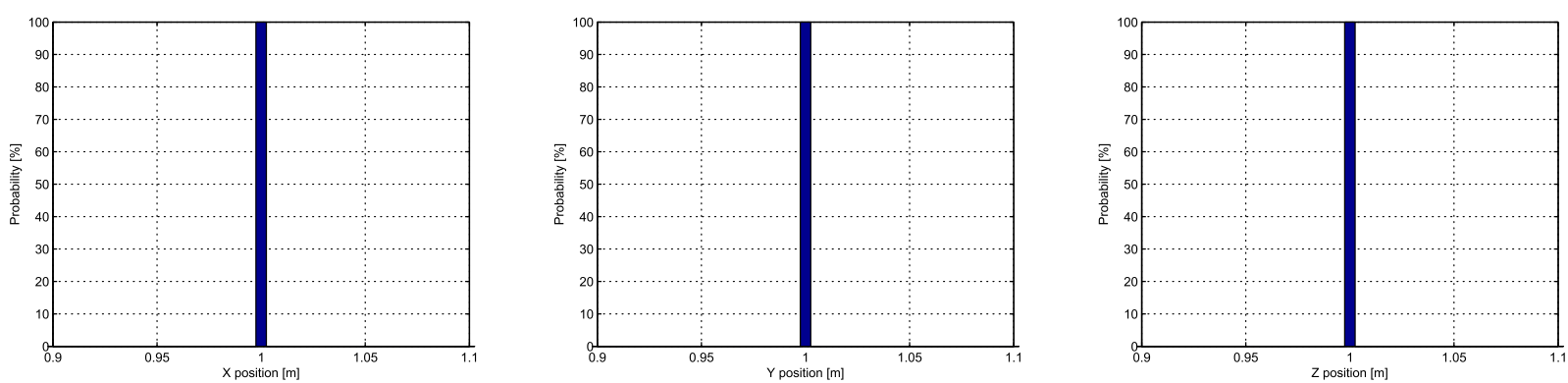

Fig. 4 Probability distributions of the determined object position at $x=y=z=1 \mathrm{~m}$.
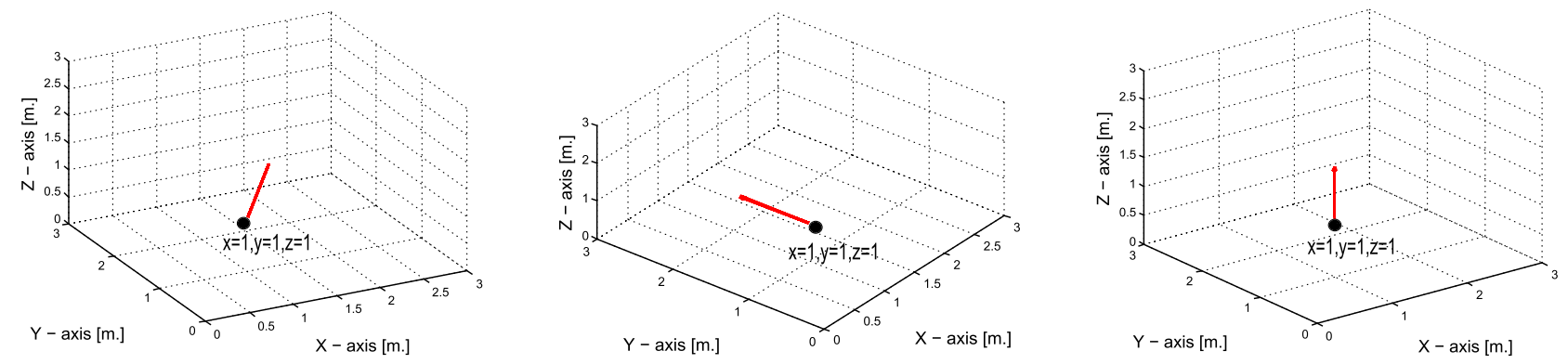

Fig. 5 Moving-object-directions estimation.

beforehand to be such that the object was moving away from the sound source in the diagonal, horizontal, and vertical lines.

\section{Conclusion}

An ultrasonic three-dimensional system for movingobject position and velocity estimation using a pair of LPM signals was proposed. The designed method comprises singlebit signal processing, Doppler-shift compensation, Taylorseries expansion, and the object-velocity-measurement formula. We employed three microphones to detect the reflected echo from the moving object. Time-of-flight and the Dopplershift relative velocity were calculated with low-cost Dopplershift compensation. Taylor-series estimation was applied as a moving-object-position calculator. Finally, the Doppler velocity and object position from the earlier measurements were used to determine the object velocity on the basis of vector theory. To confirm our idea, the moving-object position and velocity were evaluated by MATLAB computer simulation. The simulation results confirmed the validity of the proposed method.

\section{References}

[1] S. Hirata, M. K. Kurosawa and T. Katagiri, "Accuracy and resolution of ultrasonic distance measurement with high-timeresolution cross-correlation function obtained by single-bit signal processing," Acoust. Sci. \& Tech., 30, 429-438 (2009).

[2] W. Jinjin, Y. Dong and C. Ping, "Range resolution of ultrasonic distance measurement using single-bit cross correlation for robots," IEEE ICIA, pp. 917-923 (2010).

[3] J. Klahold, J. Rautenberg and U. Rckert, "Continuous sonar sensing for mobile mini-robots," IEEE IRCA, pp. 323-328 (2002).

[4] D. Marioli, E. Sardini and A. Taroni, "Ultrasonic sensing distance measurement for linear and angular position control," IEEE Trans. Instrum. Meas., 34, 578-581 (1988).

[5] S. Hirata, M. K. Kurosawa and T. Katagiri, "Cross-correlation by single-bit signal processing for ultrasonic distance measurement," IEICE Trans. Fundam. Electron. Commun. Comput. Sci., E91-A, 1031-1037 (2008).

[6] R. A. Altes, "Sonar velocity resolution with a linear period modulated pulse," J. Acoust. Soc. Am., 60 (Issue S1), S70 (1976).

[7] J. J. Kroszczynski, "Pulse compression by means of linearperiod modulation," Proc. IEEE, 57, 1260-1266 (1969).

[8] S. Hirata, M. K. Kurosawa and T. Katagiri, "Ultrasonic distance and velocity measurement by low-calculation-cost Doppler-shift compensation and high-resolution Doppler velocity estimation with wide measurement range," Acoust. Sci. \& Tech., 30, 220-223 (2009).

[9] S. Saito, M. K. Kurosawa, Y. Orino and S. Hirata, "Airbone ultrasonic position and velocity measurement using two cycles of linear-period-modulated signal," Proc. TAROS, pp. 46-53 (2011).

[10] M. Walworth and A. Mahajan, "3D Position sensing using the difference in the time-of-flights from a wave source to various recievers," Proc. ICAR, pp. 611-615 (1997).

[11] K. I. Kossonou, Y. E. Hillali, M. Bocquet, J. Assaad, A. Rivenq and I. Doumbia, "Three-dimension localization method based on time difference of arrival for ultra wide band systems," Proc. IPIN (2011).

[12] A. Pourmohammad and S. M. Ahadi "Real time high accuracy 3-D PHAT-based sound source localization using a simple 4microphone arrangement," IEEE Syst. J., 3, 455-468 (2012).

[13] K. Yu, Y. J. Guo and I. Oppermann, "Modified Taylor Series Expansion Based Positioning Algorithms," IEEE Vehicle. Tech. Conf., pp. 2656-2660 (2008).

[14] W. H. Foy, "Position-location solutions by Taylor-series estimation," IEEE Trans. Aerosp. Electron. Syst., AES-12, 187-193 (1976). 